\title{
Tool or the Teacher?-Mentoring and Mastering Using Simulation in Surgery
}

\author{
Chintamani $^{1} \cdot$ Sukriti Rastogi ${ }^{2}$ \\ Published online: 1 July 2019 \\ (C) Association of Surgeons of India 2019
}

"For the things we have to learn before we can do them, we learn by doing them".

...Aristotle

Surgery like any art form is best learned at the elbow of a mentor. The term "mentor" has its origin in the master piece Iliad and Odyssey (with the king Odysseus leaving his son with his friend Mentor to train). This concept has always been an integral part of learning and mentoring in Indian culture since ancient times. Simulation is also not new to the world. It goes back more than 3000 years when Sushruta, an Indian icon of surgery, was not only performing complex surgeries but also training his mentees in doing these procedures using innovative simulation methods (tools) like moth eaten wood for probing and watermelons and cucumber for laparotomies and fasciotomies. All trainees were expected to train for nearly 6 years and take an oath after graduation. This was very much like the Halsteadean residency and Hippocratic Oath of today [1-5].

Cambridge dictionary defines simulation as "a model of a set of problems or events that can be used to teach someone how to do something, or the process of making such a model. ...".

Simulators have an established role in aviation industry, and there is enough evidence to recommend their usage in surgery as a very effective method not only to train surgical trainees but also to assess them objectively. Many lessons have been learnt from aviation industry in using simulations to train and create various safety checklists. The strict

Chintamani

drchintamani7@gmail.com

1 Department of Surgery, Safdarjung Hospital, Vardhman Mahavir Medical College, New Delhi 110023, India

2 PGIMER, RML Hospital, New Delhi, India compliance of these checklists has shown a significant improvement in various safety-related outcomes in the operation theatres. But the art that is surgery is not just about safety [3-7].

It is an established fact that the best way to learn is to utilize one's own creativity to construct one's knowledge. Making a working model based on a thorough understanding of surgical anatomy and technique is one such way to achieve it. It is more relevant in the field of surgery because reading and understanding alone does not necessarily translate into ability to perform in a real-life scenario. Surgery still is a craft speciality that is learnt under a mentor or Guru's direct supervision. However, because of the restricted and curtailed training period and a larger number of trainees, the challenge is to create time for the trainer-trainee relationship to develop. And therefore, to maximize the training opportunities, it is necessary to explore innovative ideas to enhance the quality of training especially in surgery $[1,2,8]$.

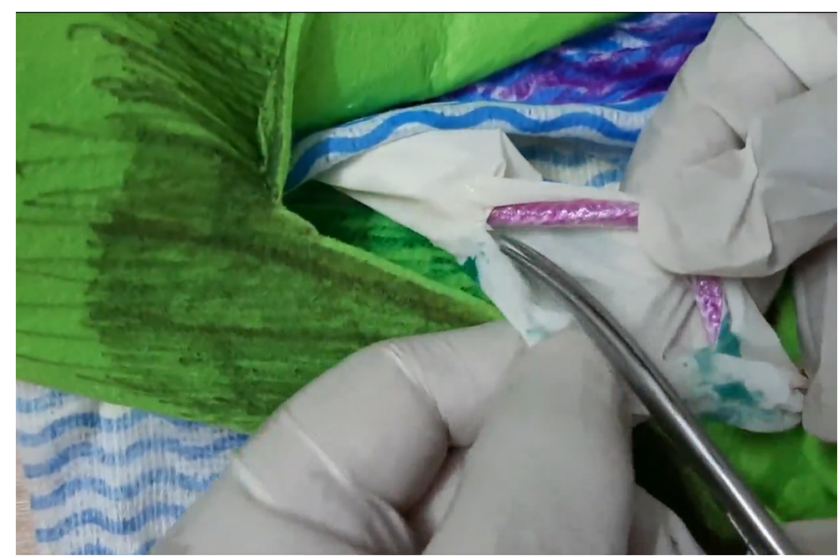

Fig. 1 Inguinal hernia: a green-coloured foam sheet depicts fibres of external oblique aponeurosis which has been incised to open the inguinal canal. The commonly available rubber glove simulating cremasteric muscle fibres, surrounding the pink-coloured rope that simulates spermatic cord 


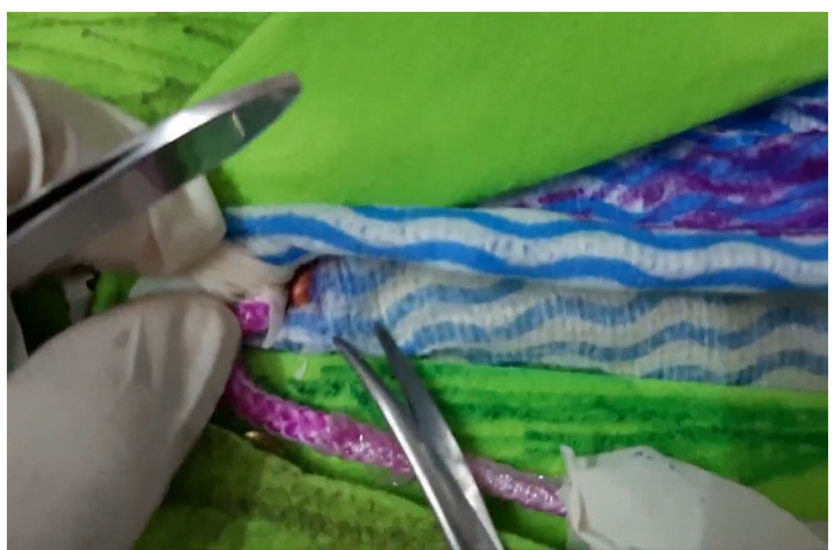

Fig. 2 The Shouldice repair for inguinal hernia: a sheet of "unwoven kitchen towel" having blue wavy lines in the image has been used for fascia transversalis and along with other layers that have been fixed to a wooden board using thumb tacks

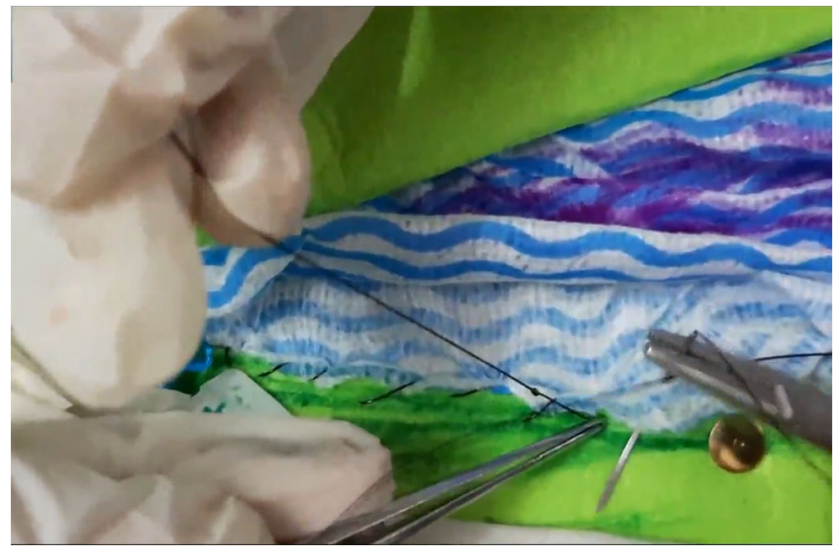

Fig. 3 The repair of the second layer by suturing the superior leaf to the inguinal ligament. Inguinal ligament has been simulated by painting the inferior aspect of the green-coloured foam sheet with a darker shade of green

Surgery as a science has perhaps outgrown the art in modern times but art still retains its place in the core. Surgeon who is a scientist has also got to be an artist before he masters the art that is surgery. The best available tools and models for

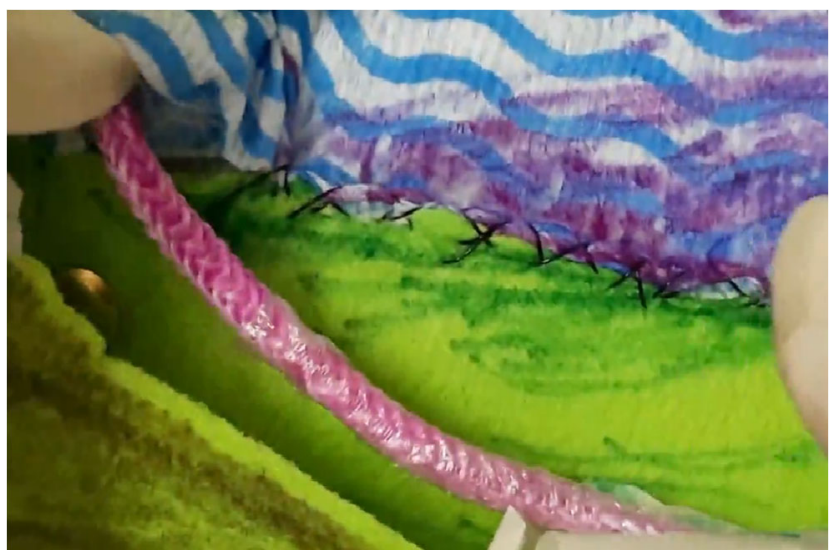

Fig. 4 The fourth and final layer demonstrating the Shouldice repair of inguinal hernia

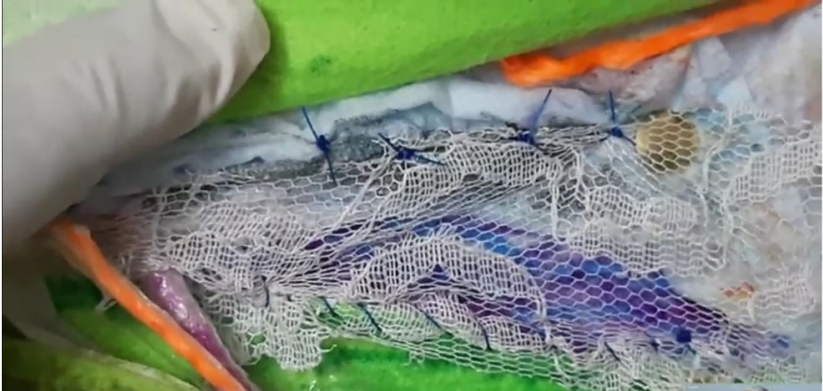

Fig. 5 Lichtenstein hernioplasty: a net cloth piece used to simulate a mesh getting fixed. The first bite is taken through the lacunar ligament

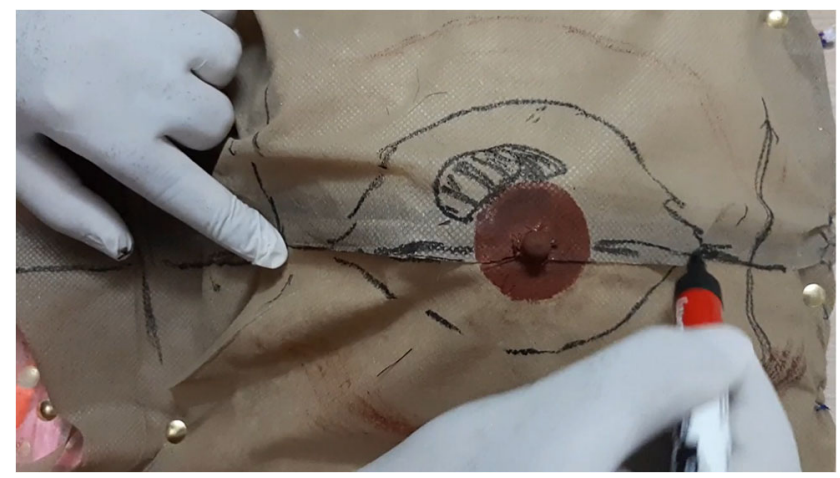

Fig. 6 Working model for demonstrating modified radical mastectomy. The figure shows a breast mount. The skin is made of a brown bag overlying a thermocol ball. Nipple areola complex is painted with brown water colour. The site of lump and incision is marked with a black marker

training that have traditionally existed over centuries include a human cadaver or animal models. But there are problems of cost and availability at all centres. Additionally, the anatomy of the dead may not truly represent a living and happening scenario.

The industry-driven and industry-created simulators that use technology are indeed very useful but again have the limitation of cost and flexibility in their usage. These softwarebased simulators do provide an almost complete simulation of

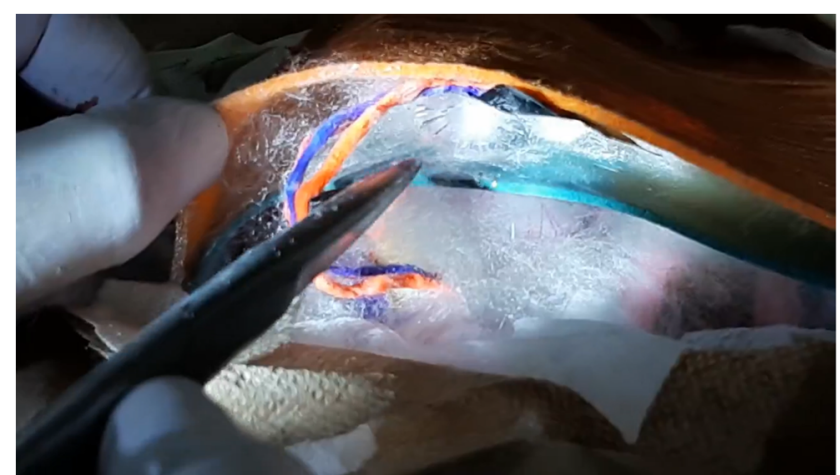

Fig. 7 Another layer of the green-coloured foam sheet fixed under the pectoralis major muscle, and it depicts the pectoralis minor. A white shiny cotton ball between the two sheets indicates Rotter's/interpectoral group of nodes 


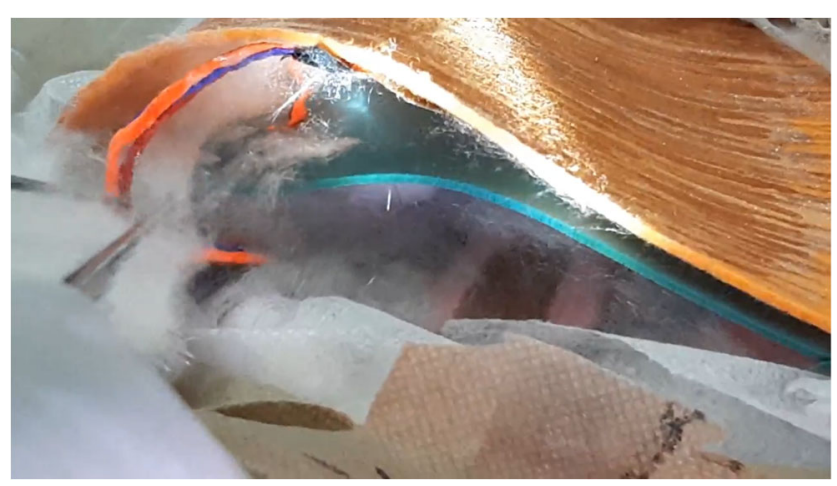

Fig. 8 Medial pectoral nerve is shown by the orange thread, supplying the P. major along with the pectoral branch of thoracoacromial artery (indicated by the red thread) and vein (indicated by the blue thread) constitute the pectoral pedicle

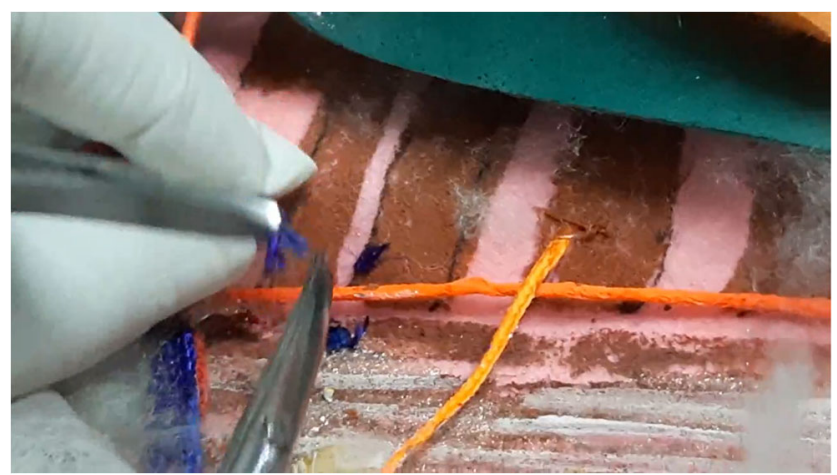

Fig. 9 Clearly seen orange thread along the chest wall demonstrates the nerve to $\mathrm{S}$. anterior

a particular surgery, but there is a limited possibility to extrapolate and use them beyond a single surgical procedure. A good mentor along with his or her mentee can create an easily reproducible teaching model based on his experience that can be tailored to demonstrate and teach various surgeries tailored to the trainees.

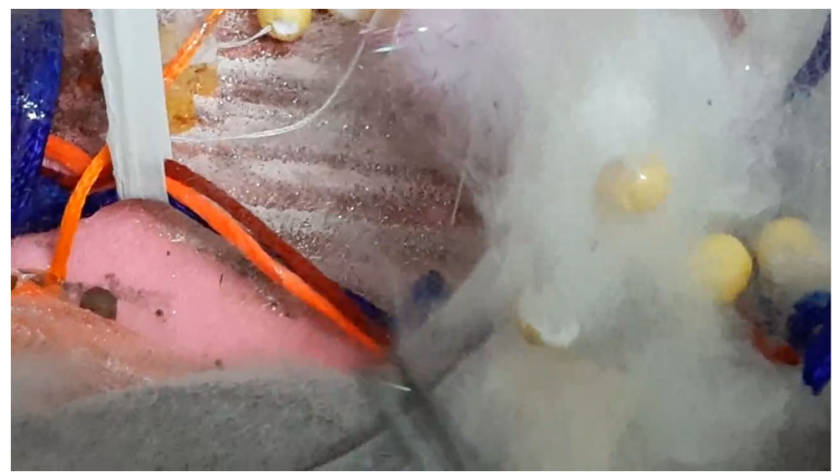

Fig. 10 Orange-, red-, and blue-coloured threads for demonstrating thoracodorsal nerve, artery, and vein together constitute the thoracodorsal pedicle. The nerve is shown entering the latissimus dorsi muscle indicated by another brown-painted foam sheet

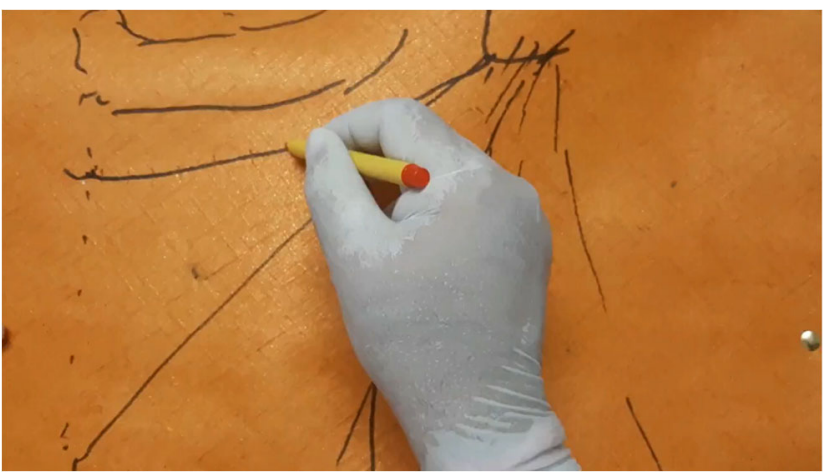

Fig. 11 Neck dissection model: a handmade sheet is used for skin, and surface marking is done with a black marker to identify the landmarks like mandible, mastoid process, SCM muscle, and trapezius. Modified Schobinger's incision is being marked in the above picture which has two limbs: horizontal and vertical

These models can be used to teach surgical anatomy of a particular region with a flexibility to demonstrate various surgeries of the region. The process of making a model can also be a very vital learning experience for the trainee. If it comes with an affordable cost and is reproducible, it can be used widely. Thus, in spite of being trainer intensive, this approach is rewarding at the end for both the mentor and the mentee.

The authors have created four models so far to demonstrate not just the anatomy of a region but also the surgical techniques to emulate a real operative scenario. These models received an encouraging feedback from both the trainers and the trainees in being very effective, reproducible, and inexpensive teaching tools. Some of these models are for learning and teaching surgeries such as inguinal hernioplasty, modified radical mastectomy, neck dissections, and laparotomy and closure of abdominal incision [Figs. 1, 2, 3, 4, 5, 6, 7, 8, 9, 10, 11, and 12].

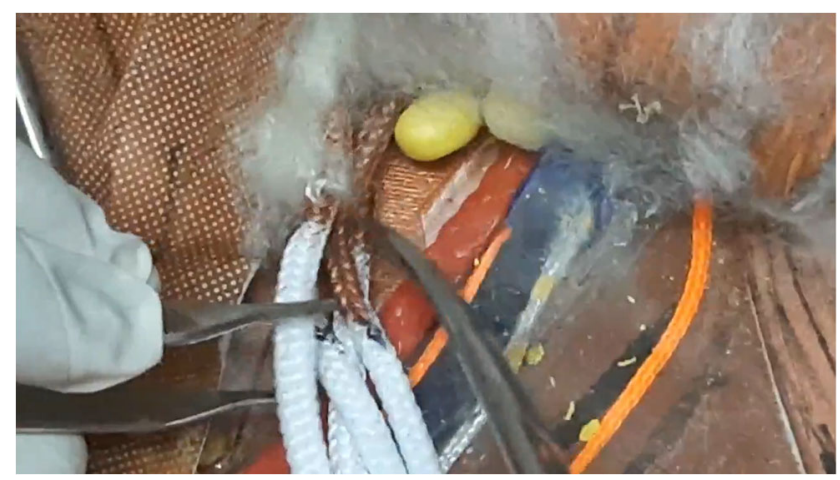

Fig. 12 Common carotid artery and internal jugular vein (IJV) demonstrated by red- and blue-coloured ropes are shown in the image. The vagus nerve is shown as an orange-coloured thread running in between the two fore-mentioned structures. Also, a transparent plastic sheet is surrounding the vessels like carotid sheath and is being dissected using dissecting scissors 
The learning and teaching of surgery needs revisiting. There is a need for expanded class rooms with a provision to provide inexpensive and easily prepared working models that can encompass complete demonstration of the steps of various commonly performed surgeries. These tools would be of special relevance for developing countries like India with a large number of trainees and limited training centres for surgery. These indigenous and inexpensive models would thus provide "Indian solutions to Indian problems". At the end of the day, it is the trainer and not just the tool and it is often quoted that "a fool with a tool is still a fool". The role of a mentor and learning by the elbow of the trainer cannot be replaced, and their significance cannot be over emphasized.

\section{References}

1. Chintamani (2015) Can making of a surgeon be a spiritual pursuit. Indian J Surg 77(5):345-346
2. Agarwal BB, Agarwal N, Chintamani DN (2018) Mentoring in surgery-Mentor, Parshuram, Dronacharya, Krishan. Indian J Surg 80(1): 81-83. https://doi.org/10.1007/s12262-017-1689-9

3. Khunger N, Kathuria S (2016) Mastering surgical skills through simulation-based learning: practice makes one perfect. J Cutan Aesthet Surg 9(1):27-31

4. Torkington J, Smith SG, Rees BI, Darzi A (2000) The role of simulation in surgical training. Ann R Coll Surg Engl 82:88-94

5. Sarker SK1, Patel B (2007) Simulation and surgical training. Int J Clin Pract 61:2120-2121

6. Sarker SK1, Patel B (2007) Simulation and surgical training. Int J Clin Pract 61:2120-2121

7. Denadai R, Toledo AP, Martinhão Souto LR (2012) Basic plastic surgery skills training program on inanimate bench models during medical graduation. Plast Surg Int 2012: 651863

8. Reznick RK, MacRae H (2006) Teaching surgical skills-changes in the wind. N Engl J Med 355:2664-2669

Publisher's Note Springer Nature remains neutral with regard to jurisdictional claims in published maps and institutional affiliations. 